\title{
CONDITIONS FOR THE UNIVALENCE OF CERTAIN INTEGRAL OPERATORS
}

\author{
R. AGHALARY, A. EBADIAN AND P. ARJOMANDINIA
}

\begin{abstract}
In the present paper, we consider two integral operators defined on special subclass of $\mathscr{A}$, namely $U_{n}(\lambda, \mu)$ with $n \in \mathbb{N}$. Using the extension of Becker's lemma, we obtain new univalence criterions for the operators in the open unit disk $\mathbb{D}=\{z \in \mathbb{C}:|z|<$ 1\}. Finally, we deduce some corollaries of the main results.
\end{abstract}

\section{Introduction}

Let $H(\mathbb{D})$ denote the class of all analytic functions in $\mathbb{D}$ and $\mathscr{A}_{n}$ be the subclass of $H(\mathbb{D})$ containing the functions $f$ of the form

$$
f(z)=z+a_{n+1} z^{n+1}+\cdots,
$$

with $\mathscr{A}_{1}=\mathscr{A}$. Suppose that $S \subseteq \mathscr{A}$ denote the class of normalized analytic and univalent functions in $\mathbb{D}$. For fixed real numbers $\lambda$ and $\mu$ with $0<\lambda \leq 1$ and $\mu(1+\lambda)<n$ consider the class $U_{n}(\lambda, \mu)$ defined by

$$
U_{n}(\lambda, \mu)=\left\{f \in \mathscr{A}_{n}:\left|\left(\frac{z}{f(z)}\right)^{\mu+1} f^{\prime}(z)-1\right|<\lambda, z \in \mathbb{D}\right\},
$$

such that $f(z) \neq 0$ for $z \in \mathbb{D} \backslash\{0\}$. For $n=1$, we have $U_{n}(\lambda, \mu)=U(\lambda, \mu)$, where the class $U(\lambda, \mu)$ was defined and studied by authors in [1,7]. For more details about $U_{n}(\lambda, \mu)$ see [8]. Now, consider the following integral operator

$$
F_{\alpha, \beta}(z)=\left\{\beta \int_{0}^{z} t^{\beta-1} \prod_{i=1}^{m}\left(\frac{f_{i}(t)}{t}\right)^{\alpha_{i}} d t\right\}^{\frac{1}{\beta}} ;\left(\alpha_{i} \in \mathbb{C}, z \in \mathbb{D}, i=1, \ldots, m\right),
$$

where $f_{i} \in \mathscr{A}, \beta \in \mathbb{C}, \Re(\beta)>0, \alpha=\left(\alpha_{1}, \ldots, \alpha_{m}\right)$ and $f_{i}(z) / z \neq 0$ for $i=1, \ldots, m$. Note that all powers in (1.2) are principal ones. This integral operator was introduced and studied by Breaz [2].

Received July 18, 2012, accepted November 13, 2012.

Communicated by Chung-Tsun Shieh.

2010 Mathematics Subject Classification. Primary 30C45; Secondary 30C80.

Key words and phrases. Integral operators, univalent functions.

Corresponding author: R. Aghalary. 
For $\alpha_{i}, \beta_{i} \in \mathbb{C}, \alpha=\left(\alpha_{1}, \ldots, \alpha_{m}\right), \beta=\left(\beta_{1}, \ldots, \beta_{m}\right), f_{i} \in \mathscr{A}$ and $f_{i}(z) / z \neq 0$ for all $f_{i}, i=1, \ldots, m$, consider the integral operator $G_{\alpha, \beta}(z)$ defined by

$$
G_{\alpha, \beta}(z)=\int_{0}^{z} \prod_{i=1}^{m}\left(f_{i}^{\prime}(t)\right)^{\alpha_{i}}\left(\frac{f_{i}(t)}{t}\right)^{\beta_{i}} d t ;(z \in \mathbb{D}, i=1, \ldots, m) .
$$

This integral operator was studied and used by Frasin [4].

The aim of this note is to use other methods and obtain new univalence conditions for the operators defined by (1.2) and (1.3). In order to get the purpose, we need the following lemmas.

Lemma 1.1. ([6]). Let $\beta \in \mathbb{C}$ and $\Re(\beta)>0, c \in \mathbb{C} \backslash\{-1\}$ with $|c| \leq 1$. If $f \in \mathscr{A}$ satisfies

$$
\left.|c| z\right|^{2 \beta}+\left(1-|z|^{2 \beta}\right) \frac{z f^{\prime \prime}(z)}{\beta f^{\prime}(z)} \mid \leq 1 ;(z \in \mathbb{D})
$$

then the integral operator

$$
F_{\beta}(z)=\left\{\beta \int_{0}^{z} t^{\beta-1} f^{\prime}(t) d t\right\}^{1 / \beta}
$$

is univalent in $\mathbb{D}$.

Lemma 1.2. [[5]] Let $\beta \in \mathbb{C}$ with $\Re(\beta)>0$. If $f \in \mathscr{A}$ satisfies

$$
\frac{1-|z|^{2 \Re(\beta)}}{\Re(\beta)}\left|\frac{z f^{\prime \prime}(z)}{f^{\prime}(z)}\right| \leq 1 ;(z \in \mathbb{D}),
$$

then the integral operator defined by (1.4) is univalent in $\mathbb{D}$.

\section{Main results}

We begin with the following lemma that will be used in the next theorems.

Lemma 2.1. For fixed real numbers $\lambda$ and $\mu$ with $0<\lambda \leq 1$ and $\mu(1+\lambda)<n$ let $f \in U_{n}(\lambda, \mu)$. There exists an analytic function $w(z)$ in $\mathbb{D}$, where $|w(z)|<1$ for $z \in \mathbb{D}$ and $w(0)=w^{\prime}(0)=\cdots=$ $w^{(n-1)}(0)=0$, such that

$$
\frac{z f^{\prime}(z)}{f(z)}=\frac{1+\lambda w(z)}{1-\lambda \mu \int_{0}^{1} \frac{w(t z)}{t^{\mu+1}} d t} ;(z \in \mathbb{D})
$$

Proof. For $f \in \mathscr{A}_{n}$, such that $f(z) / z \neq 0$, we can write

$$
\begin{aligned}
\left(\frac{z}{f(z)}\right)^{\mu+1} & =\frac{1}{\left(1+a_{n+1} z^{n}+\cdots\right)^{\mu+1}}=\frac{1}{1+(\mu+1) a_{n+1} z^{n}+\cdots} \\
& =1-(\mu+1) a_{n+1} z^{n}+\cdots .
\end{aligned}
$$


So, we obtain

$$
\begin{aligned}
\left(\frac{z}{f(z)}\right)^{\mu+1} f^{\prime}(z) & =1+(n-\mu) a_{n+1} z^{n}+\cdots \\
& =1+\lambda\left(\frac{(n-\mu)}{\lambda} a_{n+1} z^{n}+\cdots\right) .
\end{aligned}
$$

Therefore, by definition, for $f \in U_{n}(\lambda, \mu)$ there exists an analytic function $w(z)$ with $|w(z)|<1$ in $\mathbb{D}$ and $w(0)=w^{\prime}(0)=\ldots=w^{(n-1)}(0)=0$, such that

$$
\left(\frac{z}{f(z)}\right)^{\mu+1} f^{\prime}(z)=1+\lambda w(z) .
$$

Let $p(z)=(z / f(z))^{\mu}$, then $p$ is an analytic function in $\mathbb{D}$ and $p(0)=1$. Differentiating logaritmically from $p(z)$, we get

$$
z p^{\prime}(z)-\mu(p(z)-1)=-\mu \lambda w(z)
$$

Solving the first order differential equation (2.3) we conclude that

$$
p(z)=1-\lambda \mu \int_{0}^{1} \frac{w(t z)}{t^{\mu+1}} d t ;(z \in \mathbb{D}),
$$

or equally

$$
\left(\frac{f(z)}{z}\right)^{\mu}=\frac{1}{1-\lambda \mu \int_{0}^{1} \frac{w(t z)}{t^{\mu+1}} d t} .
$$

Using (2.2) and (2.4), finally we obtain

$$
\frac{z f^{\prime}(z)}{f(z)}=\frac{1+\lambda w(z)}{1-\lambda \mu \int_{0}^{1} \frac{w(t z)}{t^{\mu+1}} d t} ;(z \in \mathbb{D}) .
$$

This completes the proof.

Theorem 2.1. Let for all $i=1, \ldots, m, f_{i} \in U_{n}\left(\lambda_{i}, \mu_{i}\right)$ with $0<\lambda_{i} \leq 1$ and $\mu_{i}\left(1+\lambda_{i}\right)<n$. If

$$
\sum_{i=1}^{m} \lambda_{i}\left|\alpha_{i}\right|\left(\frac{n+\left|\mu_{i}\right|-\mu_{i}}{n-\left(\mu_{i}+\lambda_{i}\left|\mu_{i}\right|\right)}\right) \leq|\beta|,
$$

then the integral operator $F_{\alpha, \beta}(z)$ defined by (1.2) is univalent in $\mathbb{D}$.

Proof. To prove this, define the function

$$
\phi(z)=\int_{0}^{z} \prod_{i=1}^{m}\left(\frac{f_{i}(t)}{t}\right)^{\alpha_{i}} d t ;\left(\alpha_{i} \in \mathbb{C}, z \in \mathbb{D}, i=1, \ldots, m\right) .
$$

Then $\phi$ is analytic in $\mathbb{D}, \phi(0)=0, \phi^{\prime}(0)=1$ and

$$
\phi^{\prime}(z)=\prod_{i=1}^{m}\left(\frac{f_{i}(z)}{z}\right)^{\alpha_{i}} .
$$


From this and Lemma 2.1 we obtain

$$
\begin{aligned}
\left|\frac{z \phi^{\prime \prime}(z)}{\phi^{\prime}(z)}\right| & =\left|\sum_{i=1}^{m} \alpha_{i}\left(\frac{z f_{i}^{\prime}(z)}{f_{i}(z)}-1\right)\right| \\
& =\left|\sum_{i=1}^{m} \alpha_{i} \lambda_{i}\left(\frac{w_{i}(z)+\mu_{i} \int_{0}^{1} \frac{w_{i}(t z)}{t^{\mu_{i}+1}} d t}{1-\lambda_{i} \mu_{i} \int_{0}^{1} \frac{w_{i}(t z)}{t^{\mu_{i}+1}} d t}\right)\right| .
\end{aligned}
$$

Because $\left|w_{i}(z)\right|<1$ in $\mathbb{D}$ and $w_{i}(0)=w_{i}^{\prime}(0)=\ldots=w_{i}^{(n-1)}(0)=0$, by generalized Schwarz's lemma we have $\left|w_{i}(z)\right| \leq|z|^{n}$ for $i=1, \ldots, m$. Hence

$$
\left|\int_{0}^{1} \frac{w_{i}(t z)}{t^{\mu_{i}+1}} d t\right| \leq \int_{0}^{1} \frac{|z|^{n} t^{n}}{t^{\mu_{i}+1}} d t=\frac{|z|^{n}}{n-\mu_{i}} .
$$

The last inequality then shows that

$$
\left|\frac{w_{i}(z)+\mu_{i} \int_{0}^{1} \frac{w_{i}(t z)}{t^{\mu_{i}+1}} d t}{1-\lambda_{i} \mu_{i} \int_{0}^{1} \frac{w_{i}(t z)}{t^{\mu_{i}+1}} d t}\right| \leq \frac{|z|^{n}+\frac{\left|\mu_{i}\right||z|^{n}}{n-\mu_{i}}}{1-\frac{\lambda_{i}\left|\mu_{i}\right||z|^{n}}{n-\mu_{i}}}=\frac{\left(n+\left|\mu_{i}\right|-\mu_{i}\right)|z|^{n}}{n-\left(\mu_{i}+\lambda_{i}\left|\mu_{i}\right||z|^{n}\right)} .
$$

Set

$$
k_{i}(x)=\frac{\left(n+\left|\mu_{i}\right|-\mu_{i}\right) x^{n}}{n-\mu_{i}-\lambda_{i}\left|\mu_{i}\right| x^{n}} ;(0 \leq x<1, i=1, \ldots, m),
$$

then it is easy to see that $k_{i}^{\prime}(0)=0$ and $k_{i}^{\prime}(x)>0$ for $0<x<1$. Therefore

$$
\sup _{0 \leq x<1} k_{i}(x)=\frac{n+\left|\mu_{i}\right|-\mu_{i}}{n-\left(\mu_{i}+\lambda_{i}\left|\mu_{i}\right|\right)} ;(i=1, \ldots, m) .
$$

Now, from (2.5), (2.6) and last equality we deduce that

$$
\left|\frac{z \phi^{\prime \prime}(z)}{\phi^{\prime}(z)}\right| \leq \sum_{i=1}^{m} \lambda_{i}\left|\alpha_{i}\right|\left(\frac{n+\left|\mu_{i}\right|-\mu_{i}}{n-\left(\mu_{i}+\lambda_{i}\left|\mu_{i}\right|\right)}\right) .
$$

Finally, the assumption of theorem and inequality (2.7) show that

$$
\left|\left(1-|z|^{2 \beta}\right) \frac{z \phi^{\prime \prime}(z)}{\beta \phi^{\prime}(z)}\right| \leq \frac{1}{|\beta|} \sum_{i=1}^{m} \lambda_{i}\left|\alpha_{i}\right|\left(\frac{n+\left|\mu_{i}\right|-\mu_{i}}{n-\left(\mu_{i}+\lambda_{i}\left|\mu_{i}\right|\right)}\right) \leq 1 .
$$

Applying Lemma 1.1 for $\phi(z)$, we conclude that $F_{\alpha, \beta}(z) \in S$.

Taking $\mu_{i}=0$ and $\lambda_{i}=1$ in Theorem 2.1, we obtain the following result.

Corollary 2.1 ([9]). Let for all $i=1, \ldots, m, \alpha_{i} \in \mathbb{C}, f_{i} \in \mathscr{A}_{n}, \beta \in \mathbb{C}$ with $\Re(\beta)>0$ and $\left|\frac{z f_{i}^{\prime}(z)}{f_{i}(z)}-1\right|<$ 1. If $\alpha=\left(\alpha_{1}, \ldots, \alpha_{m}\right)$ and $\sum_{i=1}^{m}\left|\alpha_{i}\right| \leq|\beta|$, then the integral operator defined by (1.2) is univalent in $\mathbb{D}$.

Using Lemma 1.2 and the same techniques as in the proof of Theorem 2.1, we obtain another univalence condition for $F_{\alpha, \beta}(z)$. 
Corollary 2.2. Suppose that for $i=1, \ldots, m, \alpha_{i} \in \mathbb{C}, f_{i} \in U_{n}\left(\lambda_{i}, \mu_{i}\right)$, where $0<\lambda_{i} \leq 1$ and $\mu_{i}(1+$ $\left.\lambda_{i}\right)<n$. If $\beta \in \mathbb{C}$ and

$$
\Re(\beta) \geq \sum_{i=1}^{m} \lambda_{i}\left|\alpha_{i}\right|\left(\frac{n+\left|\mu_{i}\right|-\mu_{i}}{n-\left(\mu_{i}+\lambda_{i}\left|\mu_{i}\right|\right)}\right),
$$

then the integral operator defined by (1.2) belongs to $S$.

In the next theorem, we give sufficient condition for the operator $G_{\alpha, \beta}(z)$ defined by (1.3) to be univalent in $\mathbb{D}$.

Theorem 2.2. Suppose that $\alpha_{i}, \beta_{i} \in \mathbb{C}$ and $f_{i} \in U_{n}\left(\lambda_{i}, \mu_{i}\right)$ with $0<\lambda_{i} \leq 1$ and $\mu_{i}\left(1+\lambda_{i}\right)<n$ for all $i=1, \ldots, m$. If $\alpha=\left(\alpha_{1}, \ldots, \alpha_{m}\right), \beta=\left(\beta_{1}, \ldots, \beta_{m}\right)$ and

$$
\sum_{i=1}^{m} \lambda_{i}\left(2\left|\alpha_{i}\right|+\frac{\left(\left|\alpha_{i}\left(1+\mu_{i}\right)\right|+\left|\beta_{i}\right|\right)\left(n+\left|\mu_{i}\right|-\mu_{i}\right)}{n-\left(\mu_{i}+\lambda_{i}\left|\mu_{i}\right|\right)}\right) \leq 1,
$$

then the integral operator defined by (1.3) is univalent in $\mathbb{D}$.

Proof. Consider the function $\phi(z)$ by

$$
\phi(z)=G_{\alpha, \beta}(z)=\int_{0}^{z} \prod_{i=1}^{m}\left(f_{i}^{\prime}(t)\right)^{\alpha_{i}}\left(\frac{f_{i}(t)}{t}\right)^{\beta_{i}} d t .
$$

Then $\phi(0)=0, \phi^{\prime}(0)=1$ and $\phi(z) \in H(\mathbb{D})$. From (2.8) we see that

$$
\frac{z \phi^{\prime \prime}(z)}{\phi^{\prime}(z)}=\sum_{i=1}^{m}\left(\alpha_{i} \frac{z f_{i}^{\prime \prime}(z)}{f_{i}^{\prime}(z)}+\beta_{i}\left(\frac{z f_{i}^{\prime}(z)}{f_{i}(z)}-1\right)\right) .
$$

The formula (2.2) shows that for $f_{i} \in U_{n}\left(\lambda_{i}, \mu_{i}\right)$ there exists a Schwarz function $w_{i}(z)$ with $w_{i}(0)=w_{i}^{\prime}(0)=\ldots=w_{i}^{(n-1)}(0)=0$, such that

$$
\left(\frac{z}{f_{i}(z)}\right)^{\mu_{i}+1} f_{i}^{\prime}(z)=1+\lambda_{i} w_{i}(z) ;(i=1, \ldots, m) .
$$

Differentiating logaritmically from (2.9), we obtain that

$$
\frac{z f_{i}^{\prime \prime}(z)}{f_{i}^{\prime}(z)}=\left(\mu_{i}+1\right)\left(\frac{z f_{i}^{\prime}(z)}{f_{i}(z)}-1\right)+\frac{\lambda_{i} z w_{i}^{\prime}(z)}{1+\lambda_{i} w_{i}(z)} ;(i=1, \ldots, m) .
$$

The last relation shows that

$$
\frac{z \phi^{\prime \prime}(z)}{\phi^{\prime}(z)}=\sum_{i=1}^{m}\left\{\left(\alpha_{i}\left(1+\mu_{i}\right)+\beta_{i}\right)\left(\frac{z f_{i}^{\prime}(z)}{f_{i}(z)}-1\right)+\frac{\alpha_{i} \lambda_{i} z w_{i}^{\prime}(z)}{1+\lambda_{i} w_{i}(z)}\right\} .
$$

By Schwarz's lemma $\left|w_{i}(z)\right| \leq|z|^{n}$, also by Pick-Schwarz's lemma (see [3]) we have $\left|w_{i}^{\prime}(z)\right| \leq$ $\frac{1-\left|w_{i}(z)\right|^{2}}{1-|z|^{2}}$. So we deduce that

$$
\left|\frac{z w_{i}^{\prime}(z)}{1+\lambda_{i} w_{i}(z)}\right| \leq \frac{|z|}{1-\lambda_{i}\left|w_{i}(z)\right|} \frac{1-\left|w_{i}(z)\right|^{2}}{1-|z|^{2}} \leq \frac{|z|\left(1+\left|w_{i}(z)\right|\right)}{1-|z|^{2}} \leq \frac{|z|\left(1+|z|^{n}\right)}{1-|z|^{2}} .
$$


The formula (2.1) and inequality (2.10) then show that

$$
\left|\frac{z \phi^{\prime \prime}(z)}{\phi^{\prime}(z)}\right| \leq \sum_{i=1}^{m}\left\{\left(\left|\alpha_{i}\left(1+\mu_{i}\right)\right|+\left|\beta_{i}\right|\right) \lambda_{i} \frac{\left(n+\left|\mu_{i}\right|-\mu_{i}\right)|z|^{n}}{n-\left(\mu_{i}+\lambda_{i}\left|\mu_{i}\right||z|^{n}\right)}+\frac{\lambda_{i}\left|\alpha_{i}\right||z|\left(1+|z|^{n}\right)}{1-|z|^{2}}\right\} .
$$

Multiplying both sides to $\left(1-|z|^{2}\right)$, we conclude that

$$
\left(1-|z|^{2}\right)\left|\frac{z \phi^{\prime \prime}(z)}{\phi^{\prime}(z)}\right| \leq \sum_{i=1}^{m} \lambda_{i}\left(2\left|\alpha_{i}\right|+\frac{\left(\left|\alpha_{i}\left(1+\mu_{i}\right)\right|+\left|\beta_{i}\right|\right)\left(n+\left|\mu_{i}\right|-\mu_{i}\right)}{n-\left(\mu_{i}+\lambda_{i}\left|\mu_{i}\right|\right)}\right) \leq 1,
$$

by the assumption of theorem. The result now follows from Lemma 1.2.

Letting $\lambda_{i}=1, \mu_{i}=-1$ in Theorem 2.2, we obtain the following result. For the similar results see [4].

Corollary 2.3. Suppose that $f_{i} \in \mathscr{A}_{n},\left|f_{i}^{\prime}(z)-1\right|<1$ for $i=1, \ldots, m$ and

$$
\sum_{i=1}^{m}\left(2\left|\alpha_{i}\right|+\left|\beta_{i}\right|\left(1+\frac{2}{n}\right)\right) \leq 1 ;\left(\alpha_{i}, \beta_{i} \in \mathbb{C}, i=1, \ldots, m\right) .
$$

Then the integral operator $G_{\alpha, \beta}(z)$ defined by (1.3) belongs to $S$.

Finally, using the same techniques as in the proof of Theorem 2.1, we obtain the following result, but we omit the details. See [4] for the similar results.

Corollary 2.4. Let for $i=1, \ldots, m, 0<\lambda_{i} \leq 1, \mu_{i}\left(1+\lambda_{i}\right)<n$ and $f_{i} \in U_{n}\left(\lambda_{i}, \mu_{i}\right)$. If

$$
\sum_{i=1}^{m} \lambda_{i}\left|\alpha_{i}\right|\left(\frac{n+\left|\mu_{i}\right|-\mu_{i}}{n-\left(\mu_{i}+\lambda_{i}\left|\mu_{i}\right|\right)}\right)<1 ;\left(\alpha_{i} \in \mathbb{C}\right)
$$

then the integral operator

$$
\phi(z)=\int_{0}^{z} \prod_{i=1}^{m}\left(\frac{f_{i}(t)}{t}\right)^{\alpha_{i}} d t ;(z \in \mathbb{D}),
$$

is convex (univalent) function of order

$$
\gamma_{m}=1-\sum_{i=1}^{m} \lambda_{i}\left|\alpha_{i}\right|\left(\frac{n+\left|\mu_{i}\right|-\mu_{i}}{n-\left(\mu_{i}+\lambda_{i}\left|\mu_{i}\right|\right)}\right) .
$$

Proof. The inequality (2.7) shows that

$$
\left|\Re\left(\frac{z \phi^{\prime \prime}(z)}{\phi^{\prime}(z)}\right)\right| \leq 1-\gamma_{m},
$$

where $\gamma_{m}$ is given by (2.11). Therefore we have

$$
\Re\left(1+\frac{z \phi^{\prime \prime}(z)}{\phi^{\prime}(z)}\right) \geq \gamma_{m},
$$

and $\phi(z)$ is convex of order $\gamma_{m}$ (see [3]). 


\section{Acknowledgement}

The authors thank the referees for their helpful comments and suggestions to improve our manuscript.

\section{References}

[1] R. Aghalary and A. Ebadian, The pre-schwarzian derivative and nonlinear integral transforms, J. Comp. Anal. Appl., 13(2011), 771-775.

[2] D. Breaz and N. Breaz, Two integral operators, Stud. Babes-Bolyai Math., 47(2002), 13-19.

[3] P. L. Duren, Univalent Functions, Springer-Verlag, New York, 1983.

[4] B. A. Frasin, Order of convexity and univalency of general integral operator, J. Franklin Inst., 348(2011), 10131019.

[5] N. Pascu, An improvement of Becker's univalence criterion (Proceedings of the Commemorative Session Simion Stoilow, Brasov), (1987), 43-48.

[6] V. Pescar, A new generalization of Ahlfor's and Becker's criterion of univalence, Bull. Malays. Math. Soc., 19(1996), 53-54.

[7] S. Ponnusamy, Polya Schoenberg conjecture by Caratheodory functions, J. London Math. Soc., 51(1995), 93104.

[8] S. Ponnusamy and P. Sahoo, Geometric properties of certain linear integral transforms, Bull. Belg. Math. Soc. Simon Stevin, 12(2005), 95-108.

[9] N. Seenivasagan, Sufficient conditions for univalence, Appl. Math. E-notes, 8(2008), 30-35.

Department of Mathematics, Faculty of Science, Urmia University, Urmia, Iran.

E-mail: raghalary@yahoo.com

Department of Mathematics, Faculty of Science, Urmia University, Urmia, Iran.

E-mail: ebadian.ali@gmail.com

Department of Mathematics, Faculty of Science, Urmia University, Urmia, Iran.

E-mail: p.arjomandinia@gmail.com 